\title{
Mutagenic Activity of Airborne Particles in Center of Metropolitan Tokyo over the Past 20 Years
}

\author{
Osamu ENDO ${ }^{1)}$, Sumio GOTO ${ }^{1)}$, Daisuke NAKAJIMA ${ }^{2)}$ and Hidetsuru MATSUSHITA ${ }^{3)}$ \\ ${ }^{1)}$ Azabu University \\ (1-17-71 Fuchinobe, Chuo, Sagamihara, Kanagawa 252-5201, Japan) \\ ${ }^{2)}$ National Institute for Environmental Studies \\ (16-2 Onogawa, Tsukuba, Ibaraki 305-8506, Japan) \\ ${ }^{3)}$ Former University of Shizuoka \\ (52-1 Yada, Suruga, Shizuoka, Shizuoka 422-8526, Japan)
}

[Received June 18, 2015; Accepted January 12, 2016]

\begin{abstract}
Summary
To estimate the risks associated with long-term human exposure to potentially mutagenic compounds, it is important to measure long-term trends in the concentrations and activity of these compounds in the environment. Samples of total suspended particulates (TSP) have been collected upon a quartz fiber filter every 6 days for the past 20 years (1980-2002) from ambient air using a high-volume air sampler placed on the rooftop of the National Institute of Public Health building in Minato, Tokyo, Japan. The mutagenicity of dichloromethane extracts of TSP samples, which divided according to season, were assayed by Ames microsuspension assay using Salmonella strains TA100 and TA98, both with and without metabolic activation system (S9 mix). Mutagenic activities $\left(\mathrm{rev} / \mathrm{m}^{3}\right)$ were calculated from the dose-response curves of the samples and from those of reference airborne particles collected by the massive sampler. Mutagenic activity increased in the first decade (1980-1990) but slowly decreased in the second decade (1990-2002). Annual average mutagenicity in TA98 was mostly higher than in TA100. Mutagenic activity was higher in autumn and winter than in spring and summer.
\end{abstract}

Key words: Salmonella mutagenicity, Microsuspension assay, Airborne particle, Long-term trend

\section{INTRODUCTION}

Ambient air contains various chemical substances, some of which are carcinogens and/or mutagens. Humans continually take these chemical substances into the body by breathing, raising concerns over chronic effects such as lung cancer caused by long-term exposure to such substances. Therefore, it is very important when considering countermeasures against lung cancer to measure yearly variation of mutagenicity of airborne particulate extracts for clarifying human exposure to carcinogens/mutagens in suspended particles in ambient air. For measuring mutagenicity of environmental pollutants, Salmonella / microsome assay (Ames assay) has been widely used because it has some advantage with sensitivity, quantitativity, repeatability, and handling in comparison with other method ${ }^{1}$. Microsuspension assay ${ }^{2)}$, one of modifications of Ames assay, is more sensitive and needs less sample volume than original method. It is also easier to compare the test result with original method because it has same principle and employ same tester strains with original method. There have been therefore so many reports on mutagenicity of environmental pollutants with microsuspension assay ${ }^{3-10)}$. Although there was only a previous study of mutagenicity of airborne particulates over quarter century in Sapporo, a large city in Hokkaido region northern $\mathrm{Japan}^{11)}$, there was no study in Tokyo, largest city and capital of Japan.
In the present paper, the mutagenic activity of airborne particles in center of metropolitan Tokyo over the past 20 years was measured by using a sensitive method, microsuspension assay.

\section{MATERIALS AND METHODS}

\section{Reagents}

Solvents: Pesticide residue analysis grade dichloromethane (DCM; Kokusan Chemical Works Ltd., Japan) and fluorometric analysis grade dimethylsulfoxide (DMSO; Dojin Chemical Laboratory Ltd., Japan) were used.

Metabolic activation system: S9/Cofactor A set for Ames test (S9 mix; Oriental Yeast Co. Ltd., Japan) was used.

\section{Sampling and extraction}

Total suspended particulate (TSP) samples have been collected upon quartz fiber filter $(20 \times 25 \mathrm{~cm}$, Pallflex Products Co., U.S.A.) using high volume air sampler (Kimoto Electric Co., Japan) on the rooftop of former National Institute of Public Health located in the central part of Tokyo metropolitan (Shirokanedai, Minato-ku), Japan over the past 20 years (from March 1980 to February 2002 every 6 days (occasionally 4-7 days, including very few lack) for 24 hours), at a flow rate of about $1.3 \mathrm{~m}^{3} / \mathrm{min}$. The filter samples were stored in a 
deep-freezer $\left(-80^{\circ} \mathrm{C}\right)$ wrapped in aluminum foil and then in a plastic bag until extraction of organic matter. The filter samples were taken, and divided according to the seasons: spring (March, April and May), summer (June, July and August), autumn (September, October and November) and winter (December, January and February). TSP samples were collected and stored every 6 days so each group included approximately 15 daily samples. Two disks $40 \mathrm{~mm}$ in diameter were cut out from each collected filter using a belt punch, and the disks were cut into small pieces and placed into a $500 \mathrm{~mL}$ Erlenmeyer flask with a screw cap. Then, $150 \mathrm{~mL}$ of dichloromethane (Pesticide residue analysis-grade) was added to the flask and extraction was performed twice for $20 \mathrm{~min}$ each in a sonication bath. After sonication extraction, the supernatant fluid was filtered into an evaporation flask and concentrated in vacuo to about $150 \mathrm{~mL}$ with an evaporator. The volume of the condensate was measured with a $200 \mathrm{~mL}$ volumetric flask and $5 \mathrm{~mL}$ of condensate was moved to a vial for PAH analysis. The remain extraction $(195 \mathrm{~mL})$ was moved again to evaporation flask and also concentrated in vacuo to about $2 \mathrm{~mL}$ with an evaporator and the condensate was moved to a vial with a screw cap for mutagenicity test. After the solvent was removed by evaporation under a weak nitrogen gas flow, those samples were stored in a deep-freezer at $80^{\circ} \mathrm{C}$ until their mutation assay.

\section{Mutagenicity test}

The mutagenicity test was conducted by a microsuspension procedure which was a slight modification of Kado's method ${ }^{2)}$ using Salmonella typhimurium TA98 and TA100 strains (x 20 conc. bacterial solution: high-speed refrigerated centrifuge Hitachi CF15R) under the conditions of both with and without a metabolic activation system (S9 mix). The extracts were dissolved in DMSO and put into test tubes with $10,5,2.5$, and $1.25 \mu \mathrm{L}$ in duplicates for each dose. After adding $100 \mu \mathrm{L}$ of $0.1 \mathrm{M}$ sodium phosphate buffer or S9 mix, and 100 $\mu \mathrm{L}$ of concentrated bacteria, the tubes were capped and preincubated at $37^{\circ} \mathrm{C}$ with shaking. After 90 minutes of preincubation, the tubes were removed and $2.5 \mathrm{~mL}$ of molten top agar containing both $0.5 \mathrm{mM}$ biotin and $0.5 \mathrm{mM}$ histidine were added. The molten suspensions were immediately mixed with a vortex mixer and poured onto minimal glucose plates. The plates were incubated at $37^{\circ} \mathrm{C}$ in the dark for 48 hours and were counted using an automatic laser colony analyzer (Spiral System Instruments Inc., Model 500A). Mutagenic activity was calculated from the slope of the linear portion of the dose-response curve using the statistical model of least squares linear regression.

\section{RESULTS}

According to the reports of IPCS ${ }^{12-16)}$, it was suggested that interand intra-laboratory variances were existed due to bioassay conditions, such as tester strain, metabolic activation, material, technical error and so on. These reports were also suggested that complex mixture reference materials such as NIST SRMs were very useful to reduce these variances (repeatability and reproducibility) for biological study of complex environmental samples. In our present study, it was necessary to perform the assay is divided into 13 times for all samples that were made. For the purpose to reduce the variances, an independently established reference material (RM) sample which is DMSO solution of extract from airborne particles collected by massive air sampler on the rooftop of NIPH between 1997 and 2000 has been provided ${ }^{17)}$. This RM has been prepared for some research project grant awarded by Ministry of the Environment ${ }^{18)}$.

Table 1 shows mutagenic activity of RM sample and correction factor for normalization of mutagenic activity of airborne particulate samples. Correction factors were calculated from the mutagenic ac-

Table 1 Mutagenic activity of RM sample and correction factor for normalization of mutagenic activity of airborne particulate samples

\begin{tabular}{|c|c|c|c|c|c|c|c|c|}
\hline \multirow[b]{2}{*}{ Test No. } & \multicolumn{2}{|c|}{ TA100 (-S9) } & \multicolumn{2}{|c|}{ TA100 (+S9) } & \multicolumn{2}{|c|}{ TA98 $(-\mathrm{S} 9)$} & \multicolumn{2}{|c|}{ TA98 (+S9) } \\
\hline & $\mathrm{rev} / \mu \mathrm{L}$ & $\mathrm{CF}$ & $\operatorname{rev} / \mu \mathrm{L}$ & $\mathrm{CF}$ & $\mathrm{rev} / \mu \mathrm{L}$ & $\mathrm{CF}$ & $\mathrm{rev} / \mu \mathrm{L}$ & $\mathrm{CF}$ \\
\hline 1 & 15.05 & $(1.156)$ & 10.84 & $(0.795)$ & 46.06 & $(1.077)$ & 17.94 & $(1.055)$ \\
\hline 2 & 12.28 & $(0.934)$ & 16.28 & (1.194) & 46.52 & $(1.088)$ & 20.28 & (1.193) \\
\hline 3 & 13.28 & $(1.020)$ & 23.37 & (1.714) & 25.40 & $(0.594)$ & 18.21 & $(1.071)$ \\
\hline 4 & 11.16 & $(0.857)$ & 13.46 & $(0.987)$ & 21.01 & $(0.491)$ & 8.48 & $(0.499)$ \\
\hline 5 & 9.55 & $(0.733)$ & 10.34 & $(0.758)$ & 39.59 & $(0.926)$ & 15.87 & $(0.933)$ \\
\hline 6 & 11.33 & $(0.870)$ & 13.30 & $(0.976)$ & 34.84 & $(0.815)$ & 9.95 & $(0.585)$ \\
\hline 7 & 13.09 & $(1.005)$ & 12.91 & $(0.947)$ & 42.04 & $(0.983)$ & 13.19 & $(0.776)$ \\
\hline 8 & 15.38 & $(1.181)$ & 14.02 & (1.028) & 50.57 & (1.183) & 24.92 & $(1.465)$ \\
\hline 9 & 14.02 & $(1.076)$ & 11.12 & $(0.816)$ & 55.90 & (1.308) & 19.77 & $(1.163)$ \\
\hline 10 & 12.90 & $(0.990)$ & 10.36 & $(0.760)$ & 44.90 & $(1.050)$ & 22.65 & $(1.332)$ \\
\hline 11 & 15.03 & $(1.154)$ & 13.64 & $(1.001)$ & 52.35 & $(1.225)$ & 22.14 & $(1.302)$ \\
\hline 12 & 13.70 & $(1.052)$ & 12.32 & $(0.904)$ & 42.28 & $(0.989)$ & 11.50 & $(0.676)$ \\
\hline 13 & 12.55 & $(0.964)$ & 15.27 & $(1.120)$ & 54.28 & $(1.270)$ & 16.17 & $(0.951)$ \\
\hline mean $\pm s d$ & $13.02 \pm 1.69$ & $(1.000)$ & $13.63 \pm 3.45$ & $(1.000)$ & $42.75 \pm 10.55$ & $(1.000)$ & $17.01 \pm 5.09$ & $(1.000)$ \\
\hline
\end{tabular}

$\mathrm{CF}$ (correction factor) was ratio of the mutagenic activities of each assay and their averages. For example, CF of TA100 (-S9) at the test No.1 $(1.156)=15.05 / 13.02$ (mean value of 13 tests)

Negative (solvent = DMSO) control values were $226+/-35$ for TA100 (-S9), 225 +/- 39 for TA100 (+S9), 57 +/- 21 for TA98 (-S9), and $53+/-16$ for TA98 (+S9), respectively. AF2 and BaP were used as positive controls. Mutagenic activities (revertants per micro gram) of AF2 were $13000+/-1760$ for TA100 (-S9), 2360 +/- 489 for TA98 (-S9), and those of BaP were $677+/-82$ for TA100 (+S9), $82+/-29$ for TA98 (+S9), respectively 

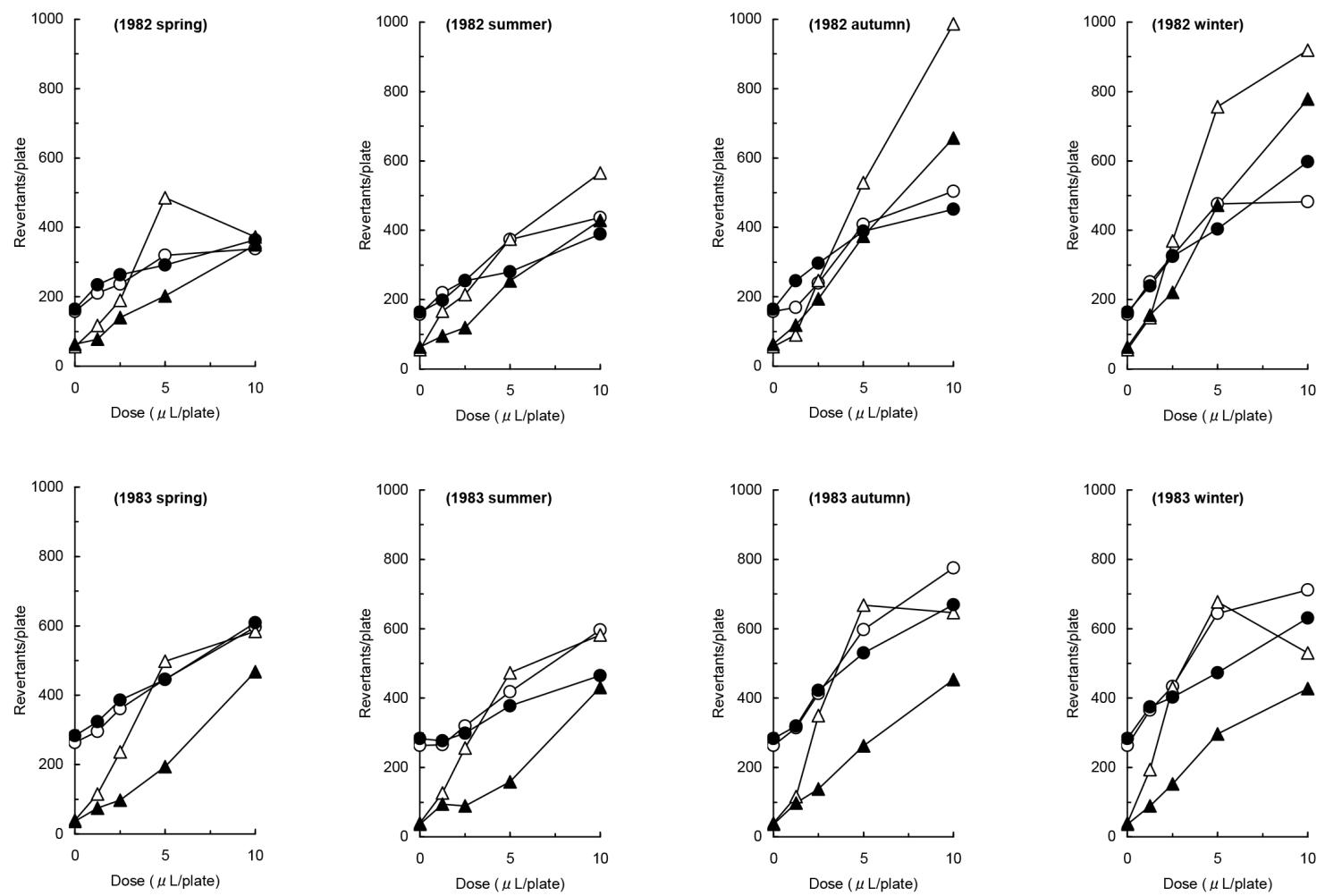

Fig. 1 Representative dose-response results for mutagenicity of airborne particulate samples by microsuspension assay - $\bigcirc$ - TA100 ( - S9) - $\triangle$ - TA98 ( - S9) - - - TA100 (+S9) - - - TA98 (+S9)

tivities of each assay and their averages. From this table, mutagenic activities (revertants per micro litter of DMSO solution, mean $+/-$ sd) of RM sample were $13.02+/-1.69$ for TA100 without S9mix, $13.63+/-3.45$ for TA100 with S9mix, $42.75+/-10.55$ for TA98 without S9mix, and $17.01+/-5.09$ for TA98 with S9mix, respectively. Relative standard deviations ranged between 13.0 and 29.9 (\%). These values were less than those of IPCS study ${ }^{16)}$. From these results, it was suggested that RM sample was very useful for correcting and normalization of the mutagenic activity of airborne particulate extracts. Thus, the correction factor of reference material provided for this study.

Figure 1 shows representative dose-response results for mutagenicity of airborne particulate samples by microsuspension assay. Every sample showed clear mutagenicty for both tester strains either with or without S9 mix. Then, the mutagenic activity was calculated from the slope of the linear portion of the dose-response curve using the statistical model of least squares linear regression and also normalized from the results of the RM sample.

Figure 2 shows yearly variation of mutagenic activity of TSP per volume of ambient air in Tokyo from 1980 to 2001. Under the condition without S9 mix, the activities for TA98 strain were higher than those for TA100 in every years. Under the condition with S9 mix, most of samples obtained similar results as above in 1980s, although some activities for TA100 were rather higher than those for TA98 after 1990. For TA100 strain, the activities have moderately decreased from year to year. It seems to be slightly higher activity without S9mix than that with S9mix in 1980s. However, after 1990, it seems to be slightly higher with S9mix than without S9mix. For TA98 strain, the mutagenic activity without S9mix decreased in mid of 1980s and have moderately decreased after 1990. The activity with S9mix has


Fig. 2 Yearly variation of mutagenic activity of TSP per volume of ambient air in Tokyo from 1980 to 2001 for strains TA100 and TA98

$\square$ without S9mix $\quad$ with S9mix 
been relatively low and has moderately decreased in every years.

Figure 3 shows long-term seasonal variation of mutagenic activities of TSP per volume of ambient air. In this figure, mutagenic activity was indicated in revertants per unit volume of air (revertants $\left./ \mathrm{m}^{3}\right)$. And also open square $(\square)$, open rhombus $(\diamond)$, closed square $(\square)$, and closed rhombus $(\diamond)$ indicate spring, summer, autumn, and winter respectively. From the figure, a clear seasonal variation was obtained in 1980s without regards to tester strains either with or without S9mix. Seasonal variation has become smaller after 1990. Generally, the activities in autumn-winter were higher than those in spring-summer. Moreover, the activities in autumn-winter season have dramatically decreased regardless to tester strains either with or without S9mix, although those in spring-summer season have moder- ately decreased for TA98 strain.

Figure 4 shows yearly variation of mutagenic activity of TSP per weight of TSP in Tokyo from 1980 to 2001. From the figure, the mutagenic activity for TA98 without S9mix has shown the maximum in 1983 and almost decreased in years after. However the activities for TA100 strain (both with and without S9mix) and those for TA98 with S9mix have shown almost even flat status. As similar as Fig. 2, the activities for TA98 strain have been higher than those for TA100 under the condition without S9 mix in every year. Under the condition with S9 mix, most of samples have obtained similar results in 1980s, although some activities for TA100 have rather been higher than those for TA98 after 1990.

Figure 5 shows long-term seasonal variation of mutagenic activi-


Fig. 3 Long-term seasonal variation of mutagenic activities of TSP per volume of ambient air

$$
\text { - } \square \text { - spring }-\diamond \text {-summer - } \mathbf{\square} \text { - autumn } \quad-\diamond \text { - winter }
$$


ties of TSP per weight of TSP. In this figure, the activity was indicated in revertants per unit weight of particle (revertants/mg, particle). The legends were as same as Fig. 3. From the figure, as similar as Fig. 3, a clear seasonal variation was obtained without regards to tester strains and with/without S9mix. However, the variation has not always decreased after 1990. The activities in autumn-winter were higher than those in spring-summer regardless to tester strains either with or without S9mix. Overall, seasonal variation of mutagenic activity per unit weight of particle was larger than that per unit volume of air. This reason is not clear currently. These result suggested that not only TSP concentration decreased during these 20 years, but also that the generation source of the pollutant has changed.

\section{DISCUSSION}

Matsumoto et al. reported mutagenic activity of airborne particulates over quarter century (1974-2001) in Sapporo, largest city in Hokkaido region northern Japan ${ }^{11,19)}$. According to the report, mutagenicity and $\mathrm{BaP}$ concentrations showed seasonal fluctuations, highest in winter, lowest in summer, reflecting fuel consumption and meteorological factors. On the other hand, Tokyo has fewer differences of the temperature than Sapporo. It was suggested that these differences of the meteorological factors reflected the difference of mutagenic aspects in Tokyo and Sapporo. Long-term trends in $\mathrm{BaP}$ indicates dramatic reduction, while indirect-acting mutagenicity showed a moderate decline and direct-acting mutagenicity showed neither a district increase nor decrease ${ }^{19,20)}$. Nitro-aromatic compounds have been increasing yearly in proportion to the number of diesel-powered vehicles. Those compounds play a major contributory role in muta-


Fig. 4 Yearly variation of mutagenic activity of TSP per weight of ambient air in Tokyo from 1980 to 2001 for strains TA100 and TA98

$\square$ without S9mix

with S9mix genic air pollution in Sapporo ${ }^{19,21)}$. Tokyo is the capital and has biggest populations in Japan. The aspects of mutagenicity such as yearly and/or seasonal variations of airborne particulates in Tokyo were very similar to those in Sapporo. From a comparison study, microsuspension assay showed about 7 times higher sensitivity than that of preincubation assay ${ }^{10}$. Our present results showed 1.34 (TA100+S9) 5.96 (TA98 - S9) times higher sensitivity than those of Matsumoto et al. in overall mean value. It seems to be a similar tendency with more considerations when it is early for ten years. It is thought that the tendency to decrease of mutagens in airborne particulates have been successful by cumulative of many pollution-abatement measures.

We have previously reported that the concentrations of PAHs decreased in past 20 years (1980-2001), especially in the late 1980s, and slowly decreased from the middle $1990 \mathrm{~s}^{22)}$. These PAHs were also higher in autumn/winter seasons than in the spring/summer seasons. These results were very similar as the report of Matsumoto et al. and as our present results of mutagenic activity. Moreover, according to the data from Tokyo Metropolitan Environmental Agency (TMEA), the concentration of sulfur dioxide, nitrogen dioxide and suspended particulate matter in the air at Shirokane station (near by our sampling point) also showed seasonal variation ${ }^{233}$.

Also according to the reports of $\mathrm{TMEA}^{23)}$, yearly average concentration of SPM had decreased from the beginning of successive measurement (fiscal year 1973) up to around 1979. Before then, although not in successive measurement, there were some difference of the concentrations of airborne particles collected with $\mathrm{HV}$ sampler by sampling points (industrial $>$ commercial $>$ residential). However it has seesawed and has been no difference by sampling points since $1980^{23,24)}$.

From our present results, it was suggested that the successive tendency to decrease of the mutagenicity of airborne particulates was caused by the effects of automobile exhaust measures and by the moves of the factories to foreign countries such as Southeast Asia areas and so on. However, it has also been suggested that take a longtime (20-30 years or more) for appearance of lung cancer by mutagens/carcinogens in air. From those facts, it was suggested that more detailed studies to chase a secular variations of the qualitative changes of the automobile exhaust with the changes of the fuel in future. Authors plan to measure the various kinds of harmful compounds such like nitro-aromatics in the specimen samples of airborne particles from now on.

\section{AKNOWLEGEMENT}

This research was partially supported by a research project grant awarded by the Azabu University.

\section{REFERENCES}

1) Yahagi, T., Nagao, M., Seino, Y., Matsushima, T., Sugimura, T. and Okada, M.: Mutagenicities of N-nitrosamines on Salmonella. Mutat. Res., 48, 121-130 (1977)

2) Kado, N.Y., Largley, D. and Eisenstadt, E.: A simple modification of the Salmonella liquid-incubation assay. Increased sensitivity for detecting mutagens in human urine. Mutat. Res., 121, 25-32 (1983)

3) Claxton, L.D., Warren, S., Zweidinger, R. and Creason, J.: A com- 

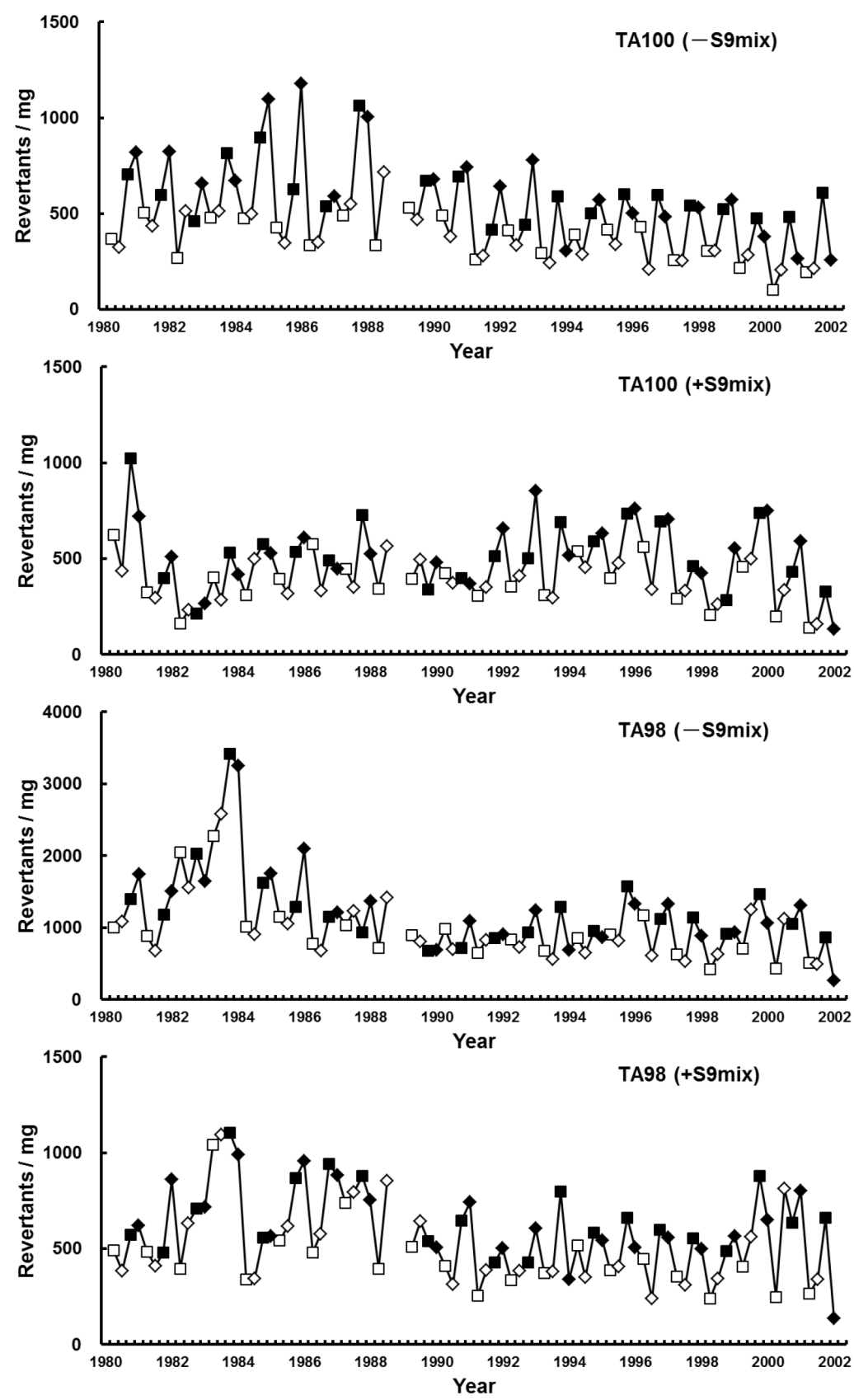

Fig. 5 Long-term seasonal variation of mutagenic activities of TSP per weight of ambient air

- $\square$ - spring - $\diamond$-summer - autumn - winter

parative assessment of Boise, Idaho, ambient air fine particle samples using the plate and microsuspension Salmonella mutagenicity assays Source. Sci. Total. Environ., 275, 95-108 (2001)

4) George, S.E., Huggins-Clark, G. and Brooks, L.R.: Use of a Salmonella microsuspension bioassay to detect the mutagenicity of munitions compounds at low concentrations. Mutat. Res., 490, 45-56 (2001)

5) Smith, C.J., Bombick, D.W., Ryan, B.A., Morgan, W.T. and Doolittle, D.J.: Urinary mutagenicity in nonsmokers following exposure to fresh diluted sidestream cigarette smoke. Mutat. Res., 470, 53-70 (2000)

6) De Martinis, B.S., Kado, N.Y., de Carvalho, L.R., Okamoto, R.A. and Gundel, L.A.: Genotoxicity of fractionated organic material in airborne particles from São Paulo, Brazil. Mutat. Res., 446, 83-94 (1999)

7) Ganesh, L., Scarlett, J.M., Lisk, D.J. and Shane, B.S.: Urinary mutagenicity as an indicator of occupational exposure in a cohort of cosmetologists. J. Toxicol. Environ. Health Part A, 57, 475-488 (1999)

8) Brooks, L.R., Hughes, T.J., Claxton, L.D., Austern, B., Brenner, R. and Kremer, F.: Bioassay-directed fractionation and chemical identification of mutagens in bioremediated soils. Environ. Health Perspect., 106, Suppl 6, 1435-40 (1998)

9) Bombick, B.R., Avalos, J.T., Nelson, P.R., Conrad, F.W. and Doolittle, J.: Comparative studies of the mutagenicity of environmental tobacco smoke from cigarettes that burn or primarily heat 
tobacco Source. Environ. Mol. Mutagen, 31, 169-175 (1998)

10) Ohkubo, T., Endo, O., Goto, S., Mineki, S., Watanabe, E. and Hayashi, T.: A study on preincubation condition for the microsuspension assay. Journal of Environmental Chemistry, 8, 841-846 (1998)

11) Matsumoto, Y., Sakai, S., Akutagawa, T., Kato, T., Akiyama, M. and Sato, H.: Mutagenicactivity of airborne particulates: A survey over a quarter century $(1974-2001)$ in Sapporo. Mutat. Res., 483 (Suppl. 1), S80 (Abstracts of $8^{\text {th }}$ International Conference on Environmental Muragens, Shizuoka, Japan) (2001)

12) Claxton, L.D., Creason, J., Leroux, B., Agurell, E., Bagley, S., Bryant, D.W., Courtois, Y.A., Douglas, G.., Clare, C.B., Goto, S., Quillardet, P., Jagannath, D.R., Kataoka, K., Mohn, G., Nielsen, P.A., Ong, T., Pederson, T.C., Shimizu, H., Nylund, L., Tokiwa, H., Vink, G.J., Wang, Y. and Warshawsky, D.: Results of IPCS collaborative study on complex mixtures. Mutat. Res., 276, 23-32 (1992)

13) Matsushita, H., Endo, O., Goto, S., Shimizu, H., Matsumoto, H., Tamakawa, K., Endo, T., Sakabe, Y., Tokiwa, H. and Ando, M.: Collaborative study using the preincubation Salmonella typhimurium mutation assay for airborne particulate matter in Japan. A trial to minimize interlaboratory variation. Mutat. Res., 271, 1-12 (1992)

14) Lewtas, J., Claxton, L.D., Rosenkrantz, H.S., Schuetzle, D., Shelby, M., Matsushita, H., Wurgler, F.E., Zimmermann, F.K., Lofroth, G., May, W.E., Krewski, D., Matsushima, T., Ohnishi, Y., Gopalan, H. N. G., Sarin, R. and Becking, G. C.: Design and implementation of a collaborative study of the mutagenicity of complex mixtures in Salmonella typhimurium. Mutat. Res., 276, 3-9 (1992)

15) Krewski, D., Leroux, B.G., Creason, J. and Claxton, L.D.: Sources of variation in the mutagenic potency of complex chemical mixtures based on the Salmonella/microsome assay. Mutat. Res.,
276, 33-59 (1992)

16) Claxton, L.D., Douglas, G., Krewski, D., Lewtas, J., Matsushita, H. and Rosenkrantz, H.S.: Overview, conclusions and recommendations of the IPCS collaborative study on complex mixtures. Mutat. Res., 276, 61-80 (1992)

17) Endo, O., Goto, S., Matsumoto, Y., Asanoma, M., Watanabe, T., Sera, N. and Wakabayashi, K.: Mutagenicity of a reference material extracted from urban air particles - A collaborative study with 5 laboratories through 3 years -. Journal of Environmental Chemistry, 11, 567-574 (2001)

18) Endo, O., Goto, S., Matsumoto, Y., Sakai, S., Akutagawa, T., Asanoma, M., Hirayama, T., Watanabe, T., Tsukatani, H., Sera, N., Tada, A. and Wakabayashi, K.: Mutagenicity of airborne particles, river water and soils in Japan from 1996 to 2003. Environ. Mutagen Res., 26, 9-22 (2004)

19) Matsumoto, Y.: A study on the mutagenic activity of airborne particulate matter - Long-term observation for past 18 years. (Thesis in Japanese)

20) Matsumoto, Y.: Variation of PAH in atmospheric environment in Japan - Hokkaido Area. Abstracts of 25th annual meeting of Japan Society of Air Pollution, 173-174 (1984)

21) Matsumoto, Y., Nakajima, T., Sakai, S., Noguchi, I. and Akiyama, M.: Seasonal variation of the mutagenic activity of airborne particulate matter in the city of cold district. Journal of the Chemical Society of Japan, 6, 837-844 (1991) (in Japanese)

22) Ezoe, Y., Goto, S., Tanabe, K., Endo, O., Koyano, M., Watanabe, I. and Matsushita, H.: Polycyclic aromatic hydrocarbon concentrations of airborne particles in urban air over the past twenty years. Polycyclic Aromatic Compounds, 24, 635-646 (2004)

23) Tokyo Metropolitan Government, Monthly monitoring data from Tokyo Metropolitan Environmental Agency, (in Japanese)

24) Japan Society for Atmospheric Environment, "History of Air Pollution in Japan vol.1", pp.78-94 (2000) (in Japanese) 